\title{
On the possibility of a bimodal solar dynamo
}

\author{
K. Petrovay ${ }^{1,2, \star}$ \\ ${ }^{1}$ Eötvös University, Department of Astronomy, Budapest, Pf. 32, H-1518 Hungary \\ ${ }^{2}$ T.I.A.R.A., Department of Physics, National Tsing Hua University, Hsinchu 30013, Taiwan
}

The dates of receipt and acceptance should be inserted later

Key words Sun: activity - Sun: magnetic fields - Sun: rotation

\begin{abstract}
A simple way to couple an interface dynamo model to a fast tachocline model is presented, under the assumption that the dynamo saturation is due to a quadratic process and that the effect of finite shear layer thickness on the dynamo wave frequency is analoguous to the effect of finite water depth on surface gravity waves. The model contains one free parameter which is fixed by the requirement that a solution should reproduce the helioseismically determined thickness of the tachocline. In this case it is found that, in addition to this solution, another steady solution exists, characterized by a four times thicker tachocline and 4-5 times weaker magnetic fields. It is tempting to relate the existence of this second solution to the occurrence of grand minima in solar activity.
\end{abstract}

Copyright line will be provided by the publisher

\section{Introduction}

The cyclically varying magnetic field that gives rise to solar activity is generally thought to arise as a consequence of an $\alpha \Omega$ dynamo mechanism. The strong toroidal field near solar maximum is a product of the solar differential rotation that winds up the weak general poloidal field. Poloidal fields are then restored by an unspecified " $\alpha$-effect", the physical meaning and spatial location of which has yet to be elucidated.

The shear associated with differential rotation is by far the strongest in a thin layer below the convective zone, known as the tachocline. Current dynamo models therefore invariably locate the $\Omega$-effect in the tachocline. The site and physical nature of the $\alpha$-effect is much less clear. As discussed in recent reviews (Petrovay 2000, Charbonneau 2005, Solanki et al. 2006), the two most widely duscussed alternatives are interface dynamos, where $\alpha$ is concentrated near the bottom of the convective zone, separated from the tachocline shear region by a thin interface only, and flux transport dynamos, where $\alpha$ is concentrated near the surface, and magnetic flux transport by meridional circulation forges a link between the surface and the tachocline.

Solar dynamo models with a jump in the diffusivity across an interface (the bottom of the convective zone) were first constructed by Ivanova and Ruzmaikin (1976, 1977). Parker (1993) developed an analytical model thas has been considered the prototype of interface dynamos ever since. Further studies of interface dynamos include Tobias (1996), Charbonneau \& MacGregor (1997), Markiel \& Thomas (1999), Petrovay \& Kerekes (2005). Flux transport dynamos were constructed, among others, by Choudhuri, Schüssler

\footnotetext{
* Corresponding author: e-mail: K.Petrovay@astro.elte.hu
}

\& Dikpati (1995), Dikpati \& Charbonneau (1999), Dikpati et al. (2004), Chatterjee, Nandy \& Choudhuri (2004).

Like the dynamo models, tachocline models also come in two main varieties. Slow tachocline models assume that the tachocline lies in the radiative interior, where turbulence is insignificant, and it is pervaded by the steady remnant magnetic field of the solar interior. If this field is limited to the radiative interior, it may be able to confine the tachocline to its observed low thickness. Whether or not such a limitation of the internal magnetic field to the radiative interior is feasible, is currently a subject of debate (Sule, Rüdiger \& Arlt 2005, Brun \& Zahn 2006).

Fast tachocline models, in contrast, assume that local instabilities and/or convective overshoot maintain a moderate level of turbulence in the tachocline. In this case the oscillatory magnetic field generated by the dynamo will penetrate the tachocline and determine its dynamics. In fact, as current dynamo models place the site of the $\Omega$-effect in the tachocline, penetration of the dynamo field in the tachocline is a necessary condition for those dynamo models to work. Following a suggestion by Gilman (2000), the first detailed models of the fast tachocline were constructed by ForgácsDajka \& Petrovay $(2001,2002)$ and further developed by Forgács-Dajka (2003).

In fast tachocline models the thickness of the tachocline is determined by the intensity of the magnetic field generated by the dynamo. The thickness of the tachocline, in turn, controls the dynamo via the shear. In this way a nonlinear coupling is expected to arise between the dynamo and a fast tachocline. In the following I will try to explore the nature of this nonlinear coupling, for concreteness focusing on the case of an interface dynamo. Section 2 describes the main features of the simple model used here, while Section 3 presents the results. Section 4 concludes the paper. 


\section{Elements of the model}

\subsection{Interface dynamo}

Parker's analytical kinematic interface dynamo (Parker 1993) consists of two semiinfinite domains (say, the halfspaces $z<0$ and $z>0$ in an xyz Cartesian frame) of diffusivity $\eta_{-}$an $\eta_{+}$, respectively. The $z>0$ domain is characterized by a constant shear $\left(\Omega=d v_{y} / d z\right)$ and no $\alpha$-effect, while the other is characterized by constant $\alpha$ and no shear. If the

$$
N=\frac{\alpha \Omega}{\eta_{-} \eta_{+} k^{3}}
$$

dynamo number is sufficiently high, a dynamo wave of form

$$
B \propto \exp \left[i k x+(\sigma+i \tilde{\omega}) \eta_{+} k^{2} t\right]
$$

is spontaneously excited at the interface (i.e. $\sigma>0$ ). In the limit $\eta_{-} / \eta_{+} \rightarrow 0$, relevant to the Sun, the dimensionless growth rate $\sigma$ and the dimensionless frequency $\tilde{\omega}$ are given by the relations

$$
\begin{aligned}
N & = \pm 8\left(\sigma+\frac{1}{2}\right)(\sigma+1)^{1 / 2} \sigma^{1 / 2} \\
& \sim \begin{cases}4 \sigma^{1 / 2} & \text { if } \sigma \ll 1 \text { (slightly supercritical case) } \\
8 \sigma^{2} & \text { if } \sigma \gg 1 \text { (strongly supercritical case) }\end{cases}
\end{aligned}
$$

and

$$
\begin{aligned}
\tilde{\omega}^{2} & =\sigma(\sigma+1) \\
& \sim \begin{cases}\sigma & \text { if } \sigma \ll 1 \text { (slightly supercritical case) } \\
\sigma^{2} & \text { if } \sigma \gg 1 \text { (strongly supercritical case) }\end{cases}
\end{aligned}
$$

In what follows we will focus on the slighly supercritical limit. Indeed: interpreting the butterfly diagram as the surface manifestation of an interface dynamo wave we find for the frequency

$$
\omega \equiv \tilde{\omega} \eta_{+} k^{2}=2 \pi / 22 \text { years } \simeq 9 \cdot 10^{-9} \mathrm{~s}^{-1} .
$$

Using $\lambda=R \pi / 2$ ( $R$ is the radius of the bottom of the convective zone) we have

$$
k=4 / R \simeq 8 \cdot 10^{-11} \mathrm{~cm}^{-1} .
$$

With a convective zone diffusivity of $\eta_{+}=6 \cdot 10^{12} \mathrm{~cm}^{2} / \mathrm{s}$ (Wang, Nash \& Sheeley 1989, Petrovay and van DrielGesztelyi 1997) this yields $\tilde{\omega} \simeq 0.23$, so $\sigma \simeq 0.05 \ll 1$. Our assumption that the slightly supercritical limit holds in the solar case is therefore justified.

\subsection{Finite shear layer}

In Parker's original model, the shear layer representing the tachocline is semi-infinite. In reality, the tachocline has some finite thickness $w$. As the difference between the rotation rate of the radiative interior and the equatorial rotation rate of the convective zone is fixed, the mean shear $\Omega$ in the tachocline, and thereby also $N$ will scale inversely with tachocline thickness: $N \propto 1 / w$. It is not known how the solutions to Parker's problem are influenced if the thickness of the shear layer is finite. In analogy to the case of surface gravity waves over shallow water here we will assume that the finite thickness of the shear layer reduces the squared mode frequency $\tilde{\omega}^{2}$ by a factor $\tanh (k w)$. (At any rate, such a factor does have the right asymptotic behavior for $w \rightarrow 0$ and $w \rightarrow \infty$, expected on physical grounds.) In the slightly subcritical case then $\sigma$ is also reduced by the same factor, so

$$
\sigma \propto N^{2} \tanh (k w) \propto \tanh (k w) / w^{2}
$$

In the solar tachocline the angular velocity is expected to relax gradually to the rigid rotation rate of the radiative interior, with some scale height $H$. Defining $w$ arbitrarily as the depth where the residual rotation has been reduced by two orders of magnitude, we have $w \sim H \ln 100$. Plugging this and equation (6) into (7) we have

$$
\sigma \propto \tanh (4 \ln 100 H / R) / H^{2}
$$

Helioseismic studies (Kosovichev 1996, Basu \& Antia 2001) indicate that at low latitude the tachocline is located immediately below the adiabatically stratified convective zone. The equatorial rotation rate relaxes to the rigid rotation rate of the radiative interior with a scale height of $H \sim 5-10 \mathrm{Mm}$ (cf. Forgács-Dajka \& Petrovay 2002); for concreteness here we will take $H=7 \mathrm{Mm}$. This shows that the tachocline is very thin indeed, its full thickness $w$ not exceeding a few percents of the solar radius. Note that at higher latitudes the tachocline may be marginally thicker, and it partly overlaps the adiabatic convective zone.

\subsection{Saturation}

Saturation of a kinematic dynamo may be brought about by a variety of effects, such as the quenching of the $\alpha$-effect for strong magnetic fields, or magnetic flux loss due to buoyancy. Without specifying the physical effect responsible for it, here we will simply describe the nonlinear saturation in a parametric form:

$$
\frac{\partial B}{\partial t}=\sigma B-a B^{1+\kappa}
$$

This implies that in the saturated state $(\partial B / \partial t=0)$ the field strength is

$$
B_{s}^{\kappa}=\sigma / a=K \tanh (4 \ln 100 H / R) / H^{2},
$$

$K$ being an undetermined amplitude.

For the nonlinearity parameter the most plausible and widely used choice is $\kappa=1$. (E.g. buoyant flux loss is expected to lead to a nonlinear term of this form.) In what follows we will therefore focus on the case $\kappa=1$. The influence of this choice of $\kappa$ on the solution will be discussed at the end of Section 3 below.

Instead of using the heuristic expressions (7)-(10), a more satisfactory approach would clearly involve a fully consistent solution of the nonlinear dynamo problem with a finite tachocline. For certain cases such solutions were presented e.g. in Chapter 10 of Zeldovich, Ruzmaikin and Sokoloff (1984). However, neither of the cases considered there ( $\alpha$-effect and shear located in two infinitesimally thin layers or in two semi-infinite layers, respectively, separated by a finite distance) does include the effect of a finite layer 
thickness. An extension of those models to the case of a finite shear layer may be a promising way to make further advance in the problem, but it is beyond the scope of the present paper.

\subsection{Fast tachocline}

The relation between the dynamo field amplitude and the tachocline scale height was derived by Forgács-Dajka \& Petrovay (2001). Let us recall the main points here. For the derivation we consider a plane parallel layer of incompressible fluid of density $\rho$, where the viscosity $\nu$ and the magnetic diffusivity $\eta$ are taken to be constant. At $z=0$ a periodic horizontal shearing flow is imposed in the $y$ direction:

$$
v_{y 0}=v_{0} \cos (k x)
$$

(so that $x$ will correspond to heliographic latitude, while $y$ to the longitude). We assume a two-dimensional flow pattern $(\partial / \partial y=0)$ and $v_{x}=v_{z}=0$. An oscillatory horizontal field is prescribed in the $x$ direction as

$$
B_{x}=B_{p} \cos (\omega t)
$$

The evolution of the azimuthal components of the velocity and the magnetic field is then described by the corresponding components of the equations of motion and induction, respectively. Introducing $v=v_{y}$ and using Alfvén speed units for the magnetic field

$$
V_{p}=B_{p}(4 \pi \rho)^{-1 / 2} \quad b=B_{y}(4 \pi \rho)^{-1 / 2},
$$

these can be written as

$$
\begin{aligned}
& \frac{\partial v}{\partial t}=V_{p} \cos (\omega t) \frac{\partial b}{\partial x}+\nu \nabla^{2} v \\
& \frac{\partial b}{\partial t}=V_{p} \cos (\omega t) \frac{\partial v}{\partial x}+\eta \nabla^{2} b
\end{aligned}
$$

Solutions may be sought in the form

$$
\begin{aligned}
& v=\bar{v}(x, z)+v^{\prime}(x, z) f(\omega t) \\
& b=b^{\prime}(x, z) f(\omega t+\phi)
\end{aligned}
$$

where $f$ is a $2 \pi$-periodic function of zero mean and of amplitude $\mathcal{O}(1)$. ( $\bar{a}$ denotes time average of $a$, while $a^{\prime} \equiv$ $a-\bar{a}$.

The (temporal) average of equation (14) reads

$$
0=V_{p} \overline{\cos (\omega t) f(\omega t+\phi)} \partial_{x} b^{\prime}-\nu \nabla^{2} \bar{v}
$$

Subtracting this from equation (14) yields

$$
\frac{\partial v^{\prime}}{\partial t}=V_{p}[\cos (\omega t) f(\omega t+\phi)]^{\prime} \frac{\partial b}{\partial x}-\nu \nabla^{2} v^{\prime}
$$

For an estimate, one can suppose $\overline{\cos (\omega t) f(\omega t+\phi)} \in$ $\mathcal{O}(1)$ (i.e. no "conspiracy" between the phases, a rather natural assumption). As $H \ll R$ we may approximate $\nabla^{2} \sim$ $H^{-2}$. Estimating the other derivatives as $\partial_{t} \sim \omega$ and $\partial_{x} \sim$ $R^{-1}$, (18) yields

$$
V_{p} b^{\prime} / R \sim \nu \bar{v} / H^{2}
$$

A similar order-of-magnitude estimate of the terms in equation (19) yields

$$
\left(\omega+\nu / H^{2}\right) v^{\prime} \sim V_{p} b^{\prime} / R
$$

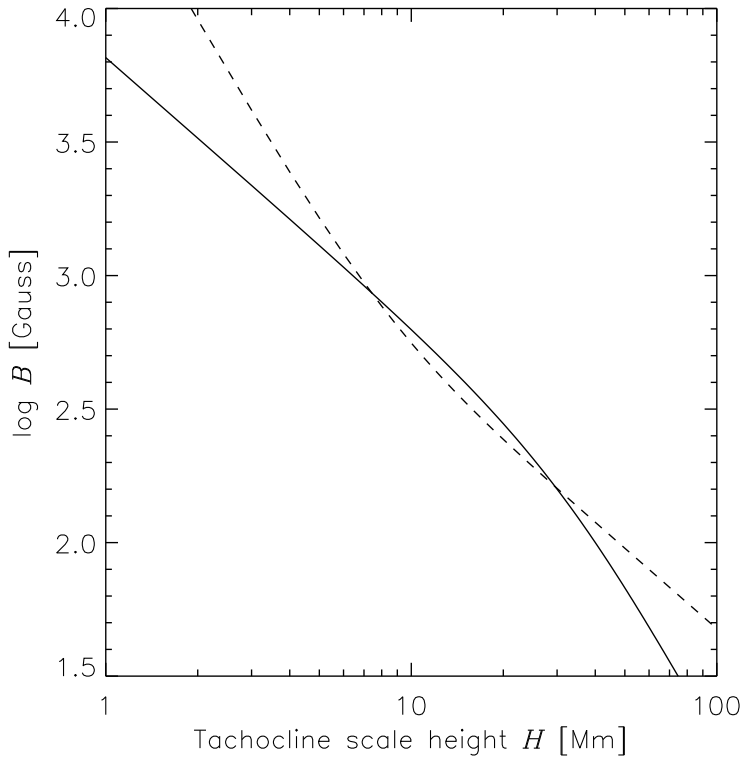

Fig. 1 Tachocline confining field strength $B_{c}$ (dashed) and dynamo saturation field strength $B_{s}$ (solid) as functions of the tachocline scale height $H$ for an interface dynamo combined with a fast tachocline. Crossing points of the curves represent steady solutions of the nonlinear dynamo problem. The undetermined amplitude of $B_{s}$ has been set so that one solution satisfies currently available seismic constraints on $H$.

while from (15) we find in a similar manner

$$
\omega b^{\prime} \sim\left(\bar{v}+v^{\prime}\right) V_{p} / R+\eta b^{\prime} / H^{2}
$$

From the last three order-of-magnitude relations one can work out with some algebra

$$
V_{p}^{2}=\frac{\nu R^{2} \omega}{H^{2}} \frac{\left(1+\eta / \omega H^{2}\right)\left(1+\nu / \omega H^{2}\right)}{1+2 \nu / \omega H^{2}}
$$

Returning from Alfvenic units to magnetic field strength we finally have

$$
B_{c}^{2}=4 \pi \rho \frac{\nu R^{2} \omega}{H^{2}} \frac{\left(1+\eta / \omega H^{2}\right)\left(1+\nu / \omega H^{2}\right)}{1+2 \nu / \omega H^{2}}
$$

For concreteness, in the numerical evaluation of equation 24 we will take the following fiducial values: $\nu=$ $\eta=10^{10} \mathrm{~cm}^{2} / \mathrm{s}, \rho=0.08 \mathrm{~g} / \mathrm{cm}^{3}$.

\section{Results}

Setting $B=B_{c}=B_{s}$, equations (10) and 24) can now be solved for $H$ and $B$. A graphical solution is presented in Figure 1. The dashed line shows relation (24) while the solid line represents the relation $(10)$. As this relation involves a free amplitude factor $K$, on this logarithmic plot we are free to shift the solid curve in the vertical direction. In the figure, the solid curve was shifted so that one crossing point falls to the value $H=7 \mathrm{Mm}$, the most likely value based on 
helioseismic constraints. In this case the mean toroidal field strength is seen to exceed one kilogauss (which does not exclude the existence of $10^{5} \mathrm{G}$ flux tubes in an intermittent field structure, cf. Ruzmaikin 2001).

It is apparent from Figure 1 that a second stationary solution also exists, with a roughly 4 to 5 times weaker mean magnetic field and a correspondingly thicker tachocline. It is tempting to identify this second solution with a "grand minimum" state of solar activity, such as the Maunder minimum of the 17th and 18th centuries. From equations (8) and (5) we expect that the dynamo period in the grand minimum mode should be about a factor of 2-2.5 longer than in normal solar activity. There is indeed some observational evidence indicating that during the Maunder minimum the dominant period in solar activity may have been around 22 years (Usoskin \& Mursula 2003), in fairly good agreement with our expectation. (Note that the present model is plane parallel, allowing a continuous mode spectrum. In a finite spherical geometry the mode spectrum will be discrete, possibly explaining why the dynamo period is exactly doubled during grand minima.)

The results presented above are valid in the case of a quadratic nonlinearity, i.e. $\kappa=1$. How does a change in the value of $\kappa$ influence the validity of the findings? Analytical considerations and numerical experimentation show that a bimodal solution persists in the range $0.5<\kappa<2$. However, for $\kappa<0.67$ the present mode of operation would correspond to the weak-field mode of the dynamo, which does not seem to agree with historical evidence. On the other hand, increasing $\kappa$ above unity, the weak-field solution becomes increasingly weaker and its tachocline depth diverges as $\kappa \rightarrow 2$. E.g. for $\kappa=1.5$ the field strength in the weak mode would be two orders of magnitude lower than presently, and the tachocline thickness would increase to $300 \mathrm{Mm}$. These values are clearly unrealistic, so the kind of bimodal solution outlined above is restricted to saturation mechanisms whose behaviour is reasonably close to quadratic.

\section{Conclusion}

Our model contains one free parameter which is fixed by the requirement that a solution should reproduce helioseismically derived thickness of the tachocline. In this case we have found that, in addition to this solution, another steady solution exists, characterized by a four times thicker tachocline and 4-5 times weaker magnetic fields. It is tempting to relate the existence this second solution to the occurrence of grand minima in solar activity.

How can the dynamo flipflop from one of these modes to the other? Stationary solutions of equation (10) are clearly linearly stable for a fixed value of $H$. Similarly, considering the full time-dependent angular momentum equation Forgács-Dajka \& Petrovay (2002) found that the fast tachocline solution is an attractor, i.e. the solution of equation
(24) for $H$ is also stable if $B_{c}$ is kept fixed. A more general stability analysis of the problem in two variables is yet to be done, but we may plausibly expect both solutions to be linearly stable if these stationary states are to be realized in the Sun for any significant period of time. Switching between the two solutions should then be due to finite amplitude stochastic disturbances. On the other hand, the possibility of a more complex nonlinear dynamical behaviour of the time-dependent system can not be discarded, with deterministic evolution between two quasi-stationary states.

The present model clearly hinges on four main assumptions. The dynamo is supposed to operate in an interface wave dominated regime (as opposed to a circulation dominated regime); the saturation mechanism is assumed to be quadratic; the effect of finite shear layer thickness on the frequency is assumed to be the same as for surface gravity waves over shallow water; and finally, the tachocline is assumed to be confined by the dynamo field. The validity of these assumptions still awaits confirmation.

Acknowledgements. This research was supported by the Theoretical Institute for Advanced Research in Astrophysics (TIARA) operated under Academia Sinica and the National Science Council Excellence Projects program in Taiwan administered through grant number NSC95-2752-M-007-006-PAE, as well as by the Hungarian Science Research Fund (OTKA) under grant no. K67746 and by the European Commission through the SOLAIRE Network (MTRN-CT-2006-035484).

\section{References}

Basu, S., Antia, H. M.: 2001, MNRAS 324, 498

Brun, A. S., Zahn, J. P.: 2006, A\&A 457, 665

Charbonneau, P.: 2005, Living Rev. Sol. Phys. 2, 2

Charbonneau, P., MacGregor, K. B.: 1997, ApJ 486, 502

Chatterjee, P., Nandy, D., Choudhuri, A. R.: 2004, A\&A 427, 1019

Choudhuri, A.R., Schüssler, M., Dikpati, M.: 1995, A\&A 303, L29

Dikpati, M., Charbonneau, P.: 1999, ApJ 518, 508

Dikpati, M., de Toma, G., Gilman, P. A., Arge, C. N., White, O. R.: 2004, ApJ 601, 1136

Forgács-Dajka, E.: 2003, A\&A 413, 1143

Forgács-Dajka, E., Petrovay, K.: 2001, Sol. Phys.203, 195

Forgács-Dajka, E., Petrovay, K.: 2002, A\&A 389, 629

Gilman, P. A.: 2000, Sol. Phys.192, 27

Ivanova, T. S., Ruzmaikin, A. A.: 1976, Soviet Astr. 20, 227

Ivanova, T. S., Ruzmaikin, A. A.: 1977, Soviet Astr. 21, 479

Kosovichev, A. G.: 1996, ApJ 469, L61

Markiel, J. A., Thomas, J. H.: 1999, ApJ 523, 827

Parker, E. N.: 1993, ApJ 408, 707

Petrovay, K.: 2000, What makes the sun tick? In The Solar Cycle and Terrestrial Climate (p. 3). ESA Publ. SP-463

Petrovay, K., Kerekes, A.: 2004, MNRAS 351, L59-L62

Petrovay, K., van Driel-Gesztelyi, L.: 1997, Sol. Phys.176, 249

Ruzmaikin, A.: 2001, Space Sci. Rev. 95, 43

Solanki, S., Inhester, B., Schüssler, M.: 2006, Rep.Prog.Phys. 69, 563

Sule, A., Rüdiger, G., Arlt, R.: 2005, A\&A 437, 1061

Tobias, S. M.: 1996, ApJ 467, 870

Usoskin, I. G., Mursula, K.: 2003, Sol. Phys.218, 319

Wang, Y. M., Nash, A. G., Sheeley, N. R.: 1989, ApJ 347, 529 
Zeldovich, Y.B., Ruzmaikin, A. A., Sokoloff, D. D.: 1984, Magnetic Fields in Astrophysics. New York: Gordon \& Breach 